\title{
Capacity and Delay Analysis for Large Social-Aware Mobile Ad Hoc Wireless Networks
}

\author{
Yang Zhou ${ }^{1, *(D)}$, Yan Shi ${ }^{1}$ and Shanzhi Chen ${ }^{2}$ \\ 1 State Key Laboratory of Networking and Switching Technology, \\ Beijing University of Posts and Telecommunications, Beijing 100876, China; shiyan@bupt.edu.cn \\ 2 State Key Laboratory of Wireless Mobile Communications, \\ China Academy of Telecommunication Technology, Beijing 100083, China; chensz@datanggroup.cn \\ * Correspondence: zhouyang_online@bupt.edu.cn
}

Received: 5 February 2020; Accepted: 26 February 2020; Published: 3 March 2020

\begin{abstract}
Most mobile ad hoc wireless networks have social features. It is a fundamental problem how to understand the performances of social-aware mobile ad hoc wireless networks. In this paper, we consider a wireless network area, with restricted mobility model and rank-based social model. On this basis, we investigate the upper bound of throughput capacity in such networks using the protocol interference model. By tessellating the network area into cells spatially and dividing time into slots temporally, we propose a multi-hop relay and slots allocation scheduling strategy. Then, we derive the achieved throughput capacity under this strategy. Results show per-node throughput is related to parameter of social model and range of node motion. In addition, we also study the delay varies by queueing theory in such network. Finally, we discuss capacity-delay tradeoffs in such networks. These results are beneficial to the design of network protocols in large social-aware mobile ad hoc wireless networks.
\end{abstract}

Keywords: mobile ad hoc wireless networks; throughput; delay; social features

\section{Introduction}

There will be trillions of devices in 5G and beyond 5G networks [1,2]. These devices are connected to the base station, then massive amounts of data will be generated. If these devices are maintained in a decentralized way, exchanging data over multi-hops (paths consisting of multiple devices), an ad hoc network is formed.

A decentralized structure that all participating devices are responsible for exchange information by themselves, makes wireless ad hoc networks useful [3]. Usually there is no previously established network infrastructure in scenarios, such as emergency services and disaster recovery. These characteristics make wireless ad hoc networks suitable for such applications where a centralized structure could be invalid.

Wireless ad hoc networks have a better scalability in contrast to centralized networks. They can be easily extended with more devices at any position in the network region. However, the mobile devices connected by radio, in which case the topology could be rapidly changed. Unpredictable mobility of nodes introduces more routing problems [4].

In this paper, we provide the scaling lows of social-aware mobile ad-hoc wireless networks. The sections are organized as follows. We introduce some related works in Section 2. In Section 3, preliminaries (such as the mobility model, social model, and throughput definitions) are introduced. In Section 4, the upper bound throughput of each node is studied. In Section 5, a multi-hop relay and slots allocation scheduling scheme is proposed, and the achieved throughput of each node is given. 
In Section 6, we investigate the network delay by queueing theory. In Section 7, we discuss and analyze the results.

\section{Related Works}

Gupta and Kumar [5,6] analyzed large wireless networks capacity from the perspective of the network layer for the first time. The wireless network is modeled as an interference network in their work. In other words, whether data is received depending on the interference between nodes. Based on this assumption, they derived the upper bound throughput of each node in random ad hoc networks with asymptotic method. However, the throughput of each node cannot reach the upper bound.

The seminal work has inspired researchers to study performances of large wireless networks. In particular, Grossglauser and Tse $[7,8]$ showed that throughput capacity changes dramatically allowing the nodes to move. A 2-hop relay algorithm was proposed with random Independent Identically Distributed Mobility Model. Using 2-hop relay algorithm, they show that the per-node throughput in large wireless networks can keep constant. But, 2-hop relay algorithm comes at the expense of latency and requires higher node speed.

Subsequently, a great deal of research has emerged on exploring performance for large wireless networks under various physical technologies and various traffic model. These include directional antennas [9], cognitive radios [10], Multiple Input Multiple Output techniques [11], etc. Multicast is a more common pattern of unicast. Li et al. $[12,13]$ studied multicast scenario with $k(k<n)$ destinations in large static wireless ad hoc networks. The results demonstrate that the upper bound throughput of each node is $\Theta\left(\frac{1}{\sqrt{n k \log n}}\right)$.

Performance of large wireless networks with more mobility models are extensively studied, such as the Brownian Mobility Model [14], Correlated Mobility Model [15], Levy Mobility Model [16], Reference Point Group Mobility Model [17], and Human Mobility Model [18].

However, high throughput and low latency cannot be obtained simultaneously. Delay can be reduced at the cost of a lower per-node throughput capacity, and vice versa. Neely and Modiano [19] analyzed this issue under different scheduling policies. The corresponding delay is $O(n), O(\sqrt{n})$ and $O(\log n)$ respectively when per-node throughput is $O(1), O\left(\frac{1}{\sqrt{n}}\right)$ and $O\left(\frac{1}{n \log n}\right)$. They showed the necessary delay and capacity tradeoffs in large wireless ad hoc network. The redundancy (flooding scheme) can decrease delay because of more transmission opportunities.

Newly added backbone nodes are beneficial for capacity and delay in mobile ad hoc networks. Data can be delivered by high-bandwidth links between backbone nodes. In Reference [20], Alexander and Veciana studied the asymptotic scaling for hybrid ad hoc networks. They analyzed the throughput capacity by dividing the number of backbone nodes into three regions. Zhang et al. [21] studied multicast traffic model impacts on capacity of hybrid networks.

Social characteristics have also become an important indicator for mobile communication network. Geographic location has a significant impact on their social relations in online social networks [22,23]. However, existing social models have not captured small world phenomenon that arbitrary nodes are likely connected through shorter friends chain. The probability of contacts decreases with the increase of distance, which obeys the power law distribution. Literature initiated the research on capacity scaling of networks with social attributes [24,25].

As the number of devices increases in large mobile ad hoc wireless networks, new challenges have to be faced in order to maintain their performance. It is of great importance how to improve the performance of such networks with social attributes. To the best of our knowledge, there are a few researches about the interaction between social networks and mobile communication networks. We are among the first attempt to study the interaction between social networks and mobile communication networks. 


\section{Preliminaries}

In this section, we want to demonstrate some models and define the problem to be solved. Some parameters used in following analysis are summarized in Table 1.

Table 1. Symbols.

\begin{tabular}{cc}
\hline Symbol & Definition \\
\hline$O$ & Asymptotic upper bound \\
$\Omega$ & Asymptotic lower bound \\
$\Theta$ & Asymptotically tight bound \\
$c, c 1, c 2, c 3$ & Constants \\
$n$ & Number of nodes \\
$W$ & Total bandwidth available \\
$r(n)$ & The transmission range \\
$m$ & Area of super-cell \\
$b(n)$ & Area of cell \\
$\delta$ & Parameter of social model \\
$\lambda_{i}$ & The input stream of source node \\
$\mathcal{T}$ & Upper bound throughput of each node \\
$\mu$ & Achieved throughput of each node \\
$D(n)$ & Network delay \\
\hline
\end{tabular}

There are $n$ mobile nodes scattering randomly over an unit square region $\mathcal{O}$, so each node is independently and uniformly distributed in size of region. We do not focus on edge effect, which is ignored in this paper. As shown in Figure 1, the network area is divided into square cells to avoid interference. We evenly divide the unit region of $n$ nodes into cells with an area $b(n)$. Let $b(n)=\Theta(\log n / n)$ to ensure connectivity in the network.

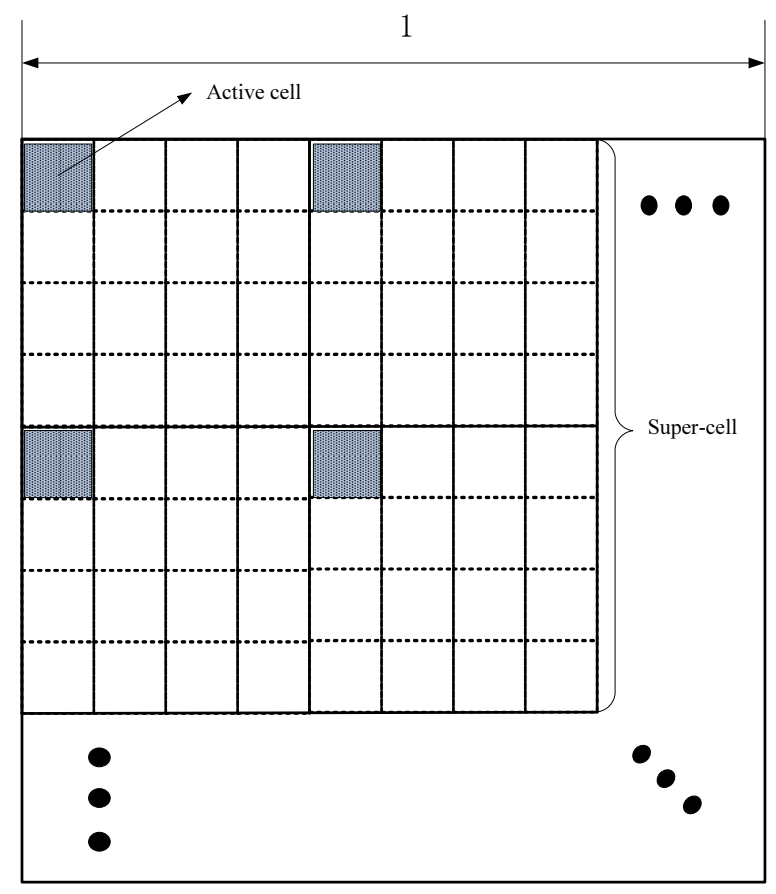

Figure 1. Network model.

\subsection{Mobility Model}

Distinct from the cell above, as shown in Figure 1, the range of node motion is restricted within the certain area, which is called super-cell. Data forward process is independent of node mobility process in the network. The area of each super-cell is $m$, so each super-cell consists of $\Theta(m / b(n))$ cells. 
There are some special cases: (1) $m=1$ the range of node motion is the whole network area, each node refresh its position in each time slot, which is similar to the Independent Identically Distributed Mobility model. (2) $m=b(n)$ the range of node motion is cell area, each node can oscillate at its initial position, which is similar to the static network.

In the super-cell, each node randomly chooses direction in $[0,2 \pi]$ and randomly chooses speed in $[0, \sqrt{m}]$. Then, we know location of nodes is independently and identically distributed in super-cell. Time is divided into slots of equal duration. At the beginning of each time slot, each node randomly selects destination cell in the super-cell, moves from its current cell to destination cell, stays in it until next time slot.

\subsection{Social Model}

We adopt the model in Reference [26] mapping from the social connections to wireless network. For a given node $i$, it select destination node $j$ based on two aspects - social experiences and geographic location. The node is likely select the destination close to it.

We define social relationship strength of node $j$ with respect to node $i$ as

$$
\operatorname{Rank}_{i}(j)=\left|\left\{k \in N: \mathcal{L}\left(X_{k}, X_{i}\right)<\mathcal{L}\left(X_{j}, X_{i}\right)\right\}\right|,
$$

where $N$ is the node set of networks, $\mathcal{L}\left(X_{k}, X_{i}\right)$ is the distance between node $k$ and node $i,|\cdot|$ represented the number of elements in the set.

The probability that node $i$ select node $j$ as a destination is defined as

$$
P_{i \rightarrow j}=\frac{1}{F_{n} \operatorname{Rank}_{i}^{\delta}(j)}
$$

where $F_{n}=\sum_{i=1}^{n} \frac{1}{i^{\delta}}$ uses for normalization.

$F_{n}$ shows the social experiences which follows the power-law degree distribution and guarantees small-world properties. $\delta$ is the parameter of social model. Notice that when $\delta=0$ and $F_{n}=0$ the network is pure mobile ad hoc network.

\subsection{Definitions}

The amount of data transmitted from the source node to the destination node is $\Lambda(t)$ in time interval $[0, t]$. Then, throughput capacity is defined as

$$
\mathcal{T}=\lim _{t \rightarrow \infty}\left(\frac{\Lambda(t)}{t}\right)
$$

From network perspective, data can be bits, bytes, or packets. If there is a spatial and temporal scheme for scheduling transmissions, the throughput of each node $\mu$ is feasible, called achieved throughput of each node.

Network delay is the total time spent by data transmission from the source node to the destination node. Since we analyze delay from the perspective of network, packet processing time of the node is neglected.

\section{The Upper Bound of Throughput Capacity}

In this section, we use the network models and assumptions above to derive an upper bound of capacity for social-aware mobile ad hoc wireless networks. For simplicity, we use S-D pairs instead of source and destination node pairs. 
Since pairs are selected by the social model, it is of great importance to find the relation between rank and geographical position. We show the relation between rank and geographical position in following lemma 1 , which is different from the destination node randomly selected pattern.

Lemma 1 ([26], Lemma 3). In large social-aware mobile ad hoc wireless networks, if the rank of node $j$ to source node $i$ is $\operatorname{Rank}_{i}(j)=r$, the distance between node $i$ and node $j$ satisfies $x=\Theta\left(\sqrt{\frac{r}{n}}\right)$ with probability 1 , when $n \rightarrow \infty$.

The probability can be calculated as

$$
\begin{aligned}
P\left(x \leq \sqrt{\frac{c r}{\pi n}}\right) & =\sum_{k=r}^{n} \frac{n !}{k !(n-k) !}\left(\frac{c r}{n}\right)^{k}\left(1-\frac{c r}{n}\right)^{n-k} \\
& <2 c_{1}\left(c e^{1-c}\right)^{r} .
\end{aligned}
$$

Similarly,

$$
P\left(x \geq \sqrt{\frac{c r}{\pi n}}\right)<2 c_{2}\left(c e^{1-c}\right)^{r} .
$$

Then, the probability $x=\Theta\left(\frac{r}{n}\right)$ can be calculated as

$$
\begin{aligned}
1-P\left(x=\Theta\left(\sqrt{\frac{r}{n}}\right)\right) & <2 c_{1}\left(c e^{1-c}\right)^{r}+2 c_{2}\left(c e^{1-c}\right)^{r} \\
& =o(1) .
\end{aligned}
$$

The whole proof of this lemma is demonstrated in Reference [26].

Lemma 2. In large social-aware mobile ad hoc wireless networks, the distance of data cumulative transmission can be represented as

$$
\mathfrak{D}= \begin{cases}\Theta\left(\frac{\sqrt{\log n}}{\sqrt{n} \sqrt{m}}\right) & 0 \leq \delta<1 \\ \Theta\left(\frac{1}{\sqrt{n \log n} \sqrt{m}}\right) & \delta=1 \\ \Theta\left(\frac{\sqrt{\log n}}{n^{\delta-1 / 2} \sqrt{m}}\right) & 1<\delta<3 / 2 \\ \Theta\left(\frac{\log ^{3 / 2} n}{n \sqrt{m}}\right) & \delta=3 / 2 \\ \Theta\left(\frac{\sqrt{\log n}}{n \sqrt{m}}\right) & \delta>3 / 2 .\end{cases}
$$

Take back the normalizing factor in (2). According to the asymptotic sum of p-series, $F_{n}$ can be represented as

$$
F_{n}=\sum_{i=1}^{n} \frac{1}{i^{\delta}}= \begin{cases}\Theta(1) & \delta>1 \\ \Theta(\log n) & \delta=1 \\ \Theta\left(n^{1-\delta}\right) & 0 \leq \delta<1\end{cases}
$$

$\mathcal{L}(S, D)$ denotes Euclidean distance of S-D pair under rank-based social model. It is clear that

$$
E\{\mathcal{L}(S, D)\}=\frac{1}{F_{n} \sqrt{n}} \sum_{r=1}^{n} \frac{\sqrt{r}}{r^{\delta}}
$$


Then,

$$
E\{\mathcal{L}(S, D)\}= \begin{cases}1 & 0 \leq \delta<1 \\ 1 / \log n & \delta=1 \\ n^{1-\delta} & 1<\delta<3 / 2 \\ \log n / \sqrt{n} & \delta=3 / 2 \\ 1 / \sqrt{n} & \delta>3 / 2\end{cases}
$$

The number of super-cells that data packets go through is $\frac{E\{\mathcal{L}(S, D)\}}{\sqrt{2 m}}$, and the average transmission distance in the cell is at least $b(n)$. The average cumulative transmitted distance $\mathfrak{D}$ can be calculated as

$$
\mathfrak{D} \geq \frac{E\{\mathcal{L}(S, D)\}}{\sqrt{2 m}} \sqrt{b(n)}
$$

Substituting $E\{\mathcal{L}(S, D)\}$ and $b(n)$, we get Lemma 2 .

Let $\mathcal{L}\left(X_{i}, X_{j}\right)$ denote the distance between node $X_{i}$ and node $X_{j}$. When the equation $\mathcal{L}\left(X_{k}, X_{i}\right) \geq$ $(1+\Delta) \mathcal{L}\left(X_{j}, X_{i}\right)$ is satisfied, data from node $i$ to node $j$ can be transmitted successfully. $\Delta$ is a given threshold to avoid interference. Euclidean distance $\mathcal{L}\left(X_{j}, X_{k}\right)$ and $\mathcal{L}\left(X_{i}, X_{l}\right)$ hold the protocol interference model, node pairs $\left(X_{i}, X_{k}\right)$ and $\left(X_{j}, X_{l}\right)$ can transmit data simultaneously.

$$
\begin{aligned}
\mathcal{L}\left(X_{j}, X_{k}\right) & \leq(1+\Delta) \mathcal{L}\left(X_{i}, X_{k}\right) \\
\mathcal{L}\left(X_{i}, X_{l}\right) & \leq(1+\Delta) \mathcal{L}\left(X_{j}, X_{l}\right) .
\end{aligned}
$$

From the triangle inequality, we have

$$
\begin{aligned}
\mathcal{L}\left(X_{j}, X_{i}\right) & \geq \mathcal{L}\left(X_{j}, X_{k}\right)-\mathcal{L}\left(X_{i}, X_{k}\right) \\
& \geq \Delta \mathcal{L}\left(X_{i}, X_{k}\right)
\end{aligned}
$$

Likewise, we have

$$
\mathcal{L}\left(X_{i}, X_{j}\right) \geq \Delta \mathcal{L}\left(X_{j}, X_{l}\right)
$$

Therefore,

$$
\mathcal{L}\left(X_{i}, X_{j}\right) \geq \frac{\Delta}{2}\left(\mathcal{L}\left(X_{i}, X_{k}\right)+\mathcal{L}\left(X_{j}, X_{l}\right)\right)
$$

Hence, there is an essential disjoint disks zone centered transmitters in the same slot, in which the radius is $\frac{\Delta}{2}$ times the length of this hop.

Square region $\mathcal{O}$ is 1 , then we have

$$
\sum_{\left(T_{i}^{t}, R_{i}^{t}\right) \in S(t)} S_{E} \leq 1
$$

where $S(t)$ is the set of transmitter-receiver pairs transmitted simultaneously. $S_{E}$ is the area of disjoint disks - consumed area that a single hop's transmission occupies. We have

$$
S_{E}=\frac{\pi \Delta^{2}}{4} \mathcal{L}\left(T_{i}^{t}, R_{i}^{t}\right)^{2}
$$

where $\mathcal{L}\left(T_{i}^{t}, R_{i}^{t}\right)$ is the distance between a transmitter-receiver pair. 
Then, we can get

$$
\sum_{\left(T_{i}^{t}, R_{i}^{t}\right) \in S(t)} \mathcal{L}\left(T_{i}^{t}, R_{i}^{t}\right)^{2} \leq \frac{4}{\pi \Delta^{2}}
$$

According to Cauchy-Schwartz inequality, we have

$$
\left(\sum_{\left(T_{i}^{t}, R_{i}^{t}\right) \in S(t)} \mathcal{L}\left(T_{i}^{t}, R_{i}^{t}\right)\right)^{2} \leq \sum_{\left(T_{i}^{t}, R_{i}^{t}\right) \in S(t)} \mathcal{L}\left(T_{i}^{t}, R_{i}^{t}\right)^{2} \sum_{\left(T_{i}^{t}, R_{i}^{t}\right) \in S(t)} 1^{2} .
$$

Assuming that all nodes are semi-duplex. $\frac{n}{2}$ transmitter-receiver node pairs can transmit at the same time, then

$$
\sum_{\left(T_{i}^{t}, R_{i}^{t}\right) \in S(t)} \mathcal{L}\left(T_{i}^{t}, R_{i}^{t}\right) \leq \sqrt{\frac{2 n}{\pi \Delta^{2}}} .
$$

During a large enough duration of time $t$, the network transport capacity is $\mathcal{T} \cdot t \cdot \frac{n}{2} \cdot \mathfrak{D}$. The amount of data transferred during a given time $t$ is at most $W \cdot t$. The network transport capacity is lesser than the product of $W \cdot t$ (the amount of data transferred) and $\sum_{\left(T_{i}^{t}, R_{i}^{t}\right) \in S(t)} \mathcal{L}\left(T_{i}^{t}, R_{i}^{t}\right)$ (the cumulative distance of all hops). Thus, the following inequality holds

$$
\begin{aligned}
\mathcal{T} \cdot t \cdot \frac{n}{2} \cdot \mathfrak{D} & \leq W \cdot t \cdot \sum_{\left(T_{i}^{t}, R_{i}^{t}\right) \in S(t)} \mathcal{L}\left(T_{i}^{t}, R_{i}^{t}\right) \\
& \leq W t \sqrt{\frac{2 n}{\pi \Delta^{2}}} .
\end{aligned}
$$

We can obtain the following inequality

$$
\mathcal{T} \leq \frac{2 \sqrt{2} \cdot W}{\Delta \mathfrak{D} \sqrt{\pi n}}
$$

Thus, an upper bound throughput of each node can be represented as

$$
\mathcal{T}=O\left(\frac{1}{\sqrt{n} \mathfrak{D}}\right) .
$$

Theorem 1. Under restricted mobility model and rank-based social model, the upper bound throughput of each node in large social-aware mobile ad hoc wireless networks is

$$
\mathcal{T}= \begin{cases}O\left(\frac{\sqrt{m}}{\sqrt{\log n}}\right) & 0 \leq \delta<1 \\ O(\sqrt{\log n} \sqrt{m}) & \delta=1 \\ O\left(\frac{n^{1-\delta} \sqrt{m}}{\sqrt{\log n}}\right) & 1<\delta<3 / 2 \\ O\left(\frac{\sqrt{n} \sqrt{m}}{\log 3 / 2}\right) & \delta=3 / 2 \\ O\left(\frac{\sqrt{n} \sqrt{m}}{\log n}\right) & \delta>3 / 2 .\end{cases}
$$

\section{Scheduling Scheme and Achieved Throughput Capacity}

In this section, we propose a multi-hop relay and slots allocation scheme, as shown in Figure 2. Then, the achieved throughput capacity based on the proposed scheme is derived. Multi-hop relay and slots allocation scheduling scheme is as follow: 


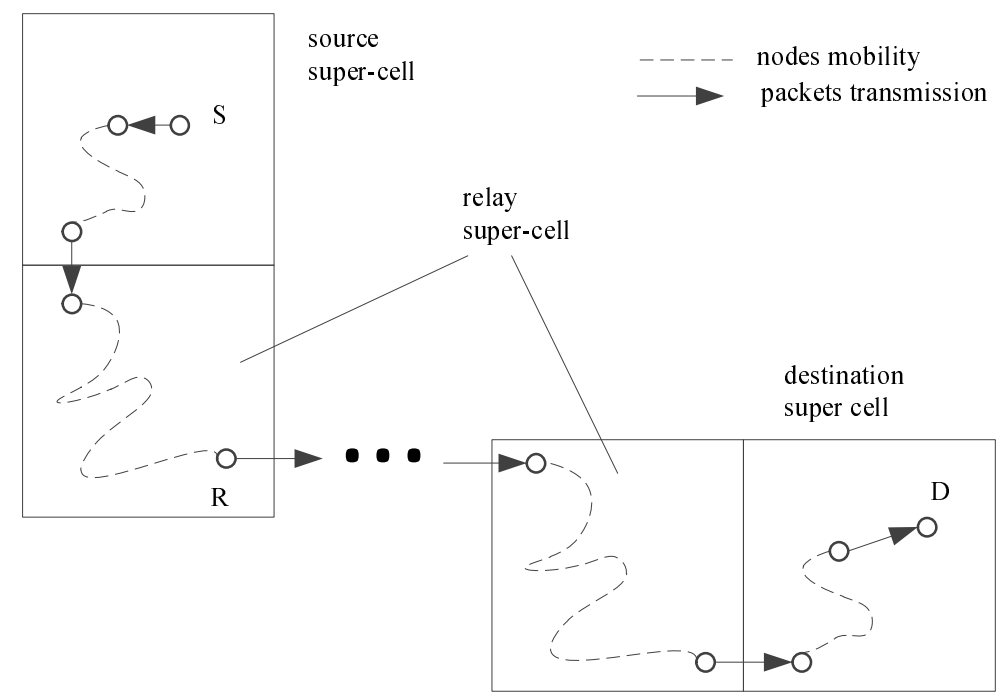

Figure 2. Multi-hop relay and slots allocation scheme.

(1) Spatially, we tessellate the network area $\mathcal{O}$ into equal square cells. A straight line segment $(\mathbb{S D}$ line) connecting the source node and the destination node denote as the reference line. Each source node forwards data approximate this $\mathrm{SD}$ line by multi-hop. Nodes in each super-cell which intersect the $\mathbb{S D}$ lines act as relay nodes.

(2) Temporally, we adopt time division multiple access (TDMA) for transmission scheduling. Time is divided into slots, all of the cells scheduled to be active in time slot sequence. Each cell becomes active in every $c^{2}(c$ is determined in the following) time slots. In an active cell, transmission is always between two nodes within the same cell or in the neighboring cells. Further, each time-slot is divided into three sub time-slots.

(3) In sub-slot A, the source node, which is located in the interior active cell, gets a transmission opportunity then randomly selects one node from in the same cell or in neighboring cells. The source node maybe transmit data to a relay node, or a destination node. If the interior active cell contains two or more source nodes, randomly select one source node. Otherwise, the interior cell remains idle.

(4) In sub-slot B, the destination node, which is located in the interior active cell, gets a transmission opportunity, then randomly selects one node from in the same cell or in neighboring cells. The transmission maybe happened between the relay node and the destination node, or maybe between the source node and destination node. If the cell contains two or more nodes, randomly select one destination node at random. Otherwise, the interior cell remains idle.

(5) In sub-slot $C$, the relay node, which is located in the border active cell, gets a transmission opportunity, then randomly selects one node from in the same cell or in neighboring cells. The transmission maybe happened between the relay node and the relay node, or maybe the relay node and the destination node. If the cell contains two or more nodes, randomly select one destination node at random. Otherwise, the border cell remains idle.

As illustrated by the shaded cells in Figure 1, active cell can transmit without interference at the same time. Let us assume that the transmission radius of each node is equal to $r(n)$. Any two active cells should have a vertical and horizontal distance of multiple of $r(n)$. Successful transmission occurs, when the following equations are satisfied.

$$
\begin{aligned}
& \mathcal{L}\left(X_{k}, X_{i}\right) \geq(1+\Delta) r(n) ; \\
& \mathcal{L}\left(X_{j}, X_{i}\right) \leq r(n) .
\end{aligned}
$$


According to multi-hop scheduling and slots allocation scheme, transmission occur when two nodes are located in the same cell or adjacent cell. So, transmission radius to be $\sqrt{8}$ times the side length of the cell $\sqrt{b(n)}$, i.e., $r(n)=\sqrt{8 b(n)}$.

Each cell alternately becomes active in $c^{2}$ time slots. The protocol interference model should be hold to guarantee the simultaneous transmissions, i.e., $c \sqrt{b(n)}=(1+\Delta) r(n)$. We obtain that $c=\sqrt{8}(1+\Delta)$. If there is at least one node in an active cell, randomly select one node to conduct transmission.

So, multi-hop scheduling that each cell becomes active in each $c^{2}$ time slots is possible. Now, we bound the number of SD lines passing through each super-cell.

Lemma 3. For each super-cell, the number of $\mathbb{S D}$ lines that multi-hop scheduling and slots allocation scheme should pass through is $O(n E\{\mathcal{L}(S, D)\} \sqrt{m})$.

Let $h_{i}$ denote the number of hops for each S-D pair, we get

$$
h_{i}=\Theta\left(\frac{E\{\mathcal{L}(S, D)\}}{\sqrt{m}}\right) .
$$

where $E\{\mathcal{L}(S, D)\}$ is the distance of S-D pairs.

The total number of hops required for all S-D pairs to send data to corresponding destination node can be represented as $N_{h}=\sum_{i=1}^{n+1} h_{i}$.

If the $k$ th hop originated from S-D pair $i$ intersects the super-cell, the random variable $I_{k}^{i}=1$, where $1 \leq i \leq n+1$ and $1 \leq k \leq h_{i}$; otherwise $I_{k}^{i}=0$. Therefore, the total number of super-cells $\mathbb{S D}$ lines passing through is $I=\sum_{1}^{n} \sum_{i=1}^{h_{i}} I_{i}^{k}$.

A given super-cell can be intersected by $\mathbb{S D}$ lines at most once. $I_{k}^{i}$ and $I_{l}^{j}$ are independent for any $1 \leq i \neq j \leq n$. Variable $I$ reflects the relaying load of a super-cell, its mathematical expectation is

$$
\begin{aligned}
E[I] & =E_{N_{h}}\left[E\left[I \mid N_{h}\right]\right] \\
& =E_{N_{h}}\left[\sum_{i=1}^{n+1} \sum_{k=1}^{h_{i}} E\left[I_{1}^{1}\right]\right] \\
& =E_{N_{h}}\left[N_{h} E\left[I_{1}^{1}\right]\right]=E_{N_{h}}\left[N_{h} m\right] \\
& =m E\left[\sum_{i=1}^{n+1} \frac{\mathcal{L}(S, D)}{\sqrt{m}}\right] \\
& =n E\{\mathcal{L}(S, D)\} \sqrt{m},
\end{aligned}
$$

where $E\left[I_{1}^{1}\right]=m$, since any hop is equally likely to originate from any of the super-cells.

Therefore, the number of super-cell that S-D lines passing through is $O(n E\{\mathcal{L}(S, D)\} \sqrt{m})$. Next, we present that the interior zone of each super-cell achieves constant per-node throughput.

Lemma 4. In large social-aware mobile ad hoc wireless networks, the per-node throughput for the interior zone of each super-cell is $\Theta(1)$, with multi-hop relay and slots allocation scheme.

Let us assume that the total number of data transmitted for each S-D pairs in sub-slot A is $D(t)$. Let $C(t)$ denote the number of cells which occur a source node transmit data to relay node transmission. Then, we have $D(t)=\sum_{1}^{n m} C(i, t)$. Since the transmission of source nodes occupied $\frac{1}{3}$ time slot, we can obtain

$$
E[D(t)]=\frac{1}{c_{1}} E[C(t)] .
$$


Next, we determine the value of $E[C(t)]$ and $E[D(t)]$. The probability that $k$ nodes are in a general cell is $P_{k}$, then

$$
P_{k}=C_{n m}^{k}\left(\frac{1}{n}\right)^{k}\left(1-\frac{1}{n}\right)^{n m-k}
$$

Obviously,

$$
\begin{aligned}
& \lim _{n \rightarrow \infty} C_{n m}^{k}\left(\frac{1}{n}\right)^{k}=\frac{1}{2^{k} k !} ; \\
& \lim _{n \rightarrow \infty}\left(1-\frac{1}{n}\right)^{n m-k}=\frac{1}{e^{m}} .
\end{aligned}
$$

Thus,

$$
\lim _{n \rightarrow \infty} P_{k}=\frac{1}{2^{k} k ! e^{m}} .
$$

The event that an source node in interior cell transmit data to relay node transmission denote by indicator $-\mathcal{I}_{i} \cdot \mathcal{I}_{i}$ contains two parts: One is the probability that cell contains exactly one source node, not a destination node represented by $P_{1}\left(1-P_{0}\right)$, The other is the probability that cell contains two or more nodes represented by $1-P_{0}-P_{1}$. Thus, we can calculate the mathematical expectation of $\mathcal{I}_{i}$,

$$
\begin{aligned}
& E\left[\mathcal{I}_{i}\right]=P_{1}\left(1-P_{0}\right)+\left(1-P_{0}-P_{1}\right) \\
& \rightarrow \frac{1}{2 e^{m}}\left(1-\frac{1}{e^{m}}\right)+\left(1-\frac{1}{e^{m}}-\frac{1}{2} \frac{1}{e^{m}}\right) \\
& =c_{2} .
\end{aligned}
$$

Therefore,

$$
\begin{aligned}
E[D(t)] & =\frac{1}{c_{1}} E[C(t)] \\
& =\frac{1}{c_{1}} E\left[\sum_{i=1}^{n m} \mathcal{I}_{i}\right] \\
& =\frac{n m}{c_{1}} E\left[\mathcal{I}_{1}\right] \\
& \rightarrow c_{3} n m,
\end{aligned}
$$

where $c_{3}=c_{2} / c_{1}$.

Each node randomly chooses direction and randomly chooses speed in super-cell. Then, we know the state can model as an irreducible finite-state Markov Chain. Therefore, over a period of time slot $N$, by the ergodicity of such a Markov Chain, we get

$$
\begin{aligned}
E[D(t)] & =\lim _{N \rightarrow \infty} \frac{1}{t} \sum_{t=1}^{N} D(t) \\
& \rightarrow c n m .
\end{aligned}
$$

Thus, the total number of data transmitted from sources node is $\Theta(n m)$, which equals to the number of nodes in super-cell. We obtain that each source node can transmit at rate of $\Theta(1)$. Similar analyses can employ in sub-slot $B$ and sub-slot $C$. Data packets are queued at the relay nodes or reach the final destination node in sub-slot $B$ and sub-slot $C$. 
Combining Lemmas 3 and 4, we get the achieved throughput of each node straightforwardly

$$
\mu=\frac{1}{n E\{\mathcal{L}(S, D)\} \sqrt{m}} .
$$

Theorem 2. Based on the multi-hop scheduling and slots allocation scheme, the achieved throughput of each node in large social-aware mobile ad hoc wireless networks can be expressed as

$$
\mu= \begin{cases}\Omega\left(\frac{1}{n \sqrt{m}}\right) & 0 \leq \delta<1 \\ \Omega\left(\frac{\log n}{n \sqrt{m}}\right) & \delta=1 \\ \Omega\left(\frac{1}{n^{2-\delta} \sqrt{m}}\right) & 1<\delta<3 / 2 \\ \Omega\left(\frac{\sqrt{n} \sqrt{m}}{\log n}\right) & \delta=3 / 2 \\ \Omega\left(\frac{1}{\sqrt{n} \sqrt{m}}\right) & \delta>3 / 2 .\end{cases}
$$

\section{Network Delay of Our Scheduling Scheme}

In this section, we provide an analysis on network delay of multi-hop relay and slots allocation scheduling scheme based on queueing theory. The delay consists of two components: hop delay (processing delay) and network delay (queuing delay). Noted that the processing time (such as coding/decoding) spent is negligible. Like many important work, hop delay is ignored in this paper.

It worth noting that the value of $E\{\mathcal{L}(S, D)\}$ is larger than value of super-cell $m$ for social parameter $0 \leq \delta \leq 3 / 2$. The S-D pairs are not located in the same super-cell. For social parameter $\delta>3 / 2$, the S-D pairs are located in the same super-cell. Thus, we solve the problem in two cases.

Let $\lambda_{i}\left(\lambda_{i}=O(\mu)\right)$ denote the input stream for source node $i$. The total rate of each node $\mu$ can be divided into two parts: $r 1$ represents the rate that the node is scheduled to transmit to the relay node; $r 2$ represents the rate that the node is scheduled to transmit to the destination node.

For social parameter $\delta \geq 3 / 2$, there are two possible routings from source node to its destination node: one is single hop from source node to destination node directly; the other is two hop path source node to relay node transmission and relay node to destination node transmission.

Since data transmission processes is independent of mobility processes, the network delay can compute from the perspective of source node $i$. Decoupled transmission path is illustrated in Figure 3 , which can be modeled as discrete queue.

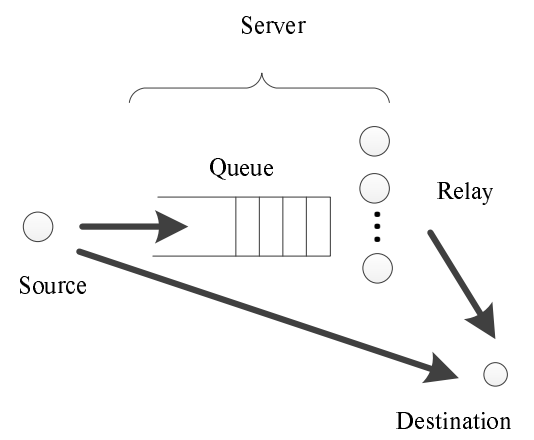

Figure 3. Queue in network for $\delta>3 / 2$.

Source node generates data at rate of $\lambda_{i}$, and service opportunity arises with some fixed rate $\mu$. Thus, source node have an expected number of data given by

$$
\bar{L}_{s}=\frac{\rho\left(1-\lambda_{i}\right)}{1-\rho},
$$

where $\rho=\frac{\lambda_{i}}{\mu}$. 
It is a reversible queue, the output stream is also a discrete stream at rate $\lambda_{i}$. Data from this output process is transmitted to relay node. Because the total number of relay nodes in each super-cell is $n m-2$, data delivered to a relay node with probability $\frac{r 1}{u}$ equally.

Hence, each relay node independently receives data with probability $\lambda_{r}=\frac{\lambda_{i} r 1}{\mu(n m-2)}$. Because the total number of relay nodes in each super-cell is $n m-2$, the relay node is scheduled for data transmission to the destination node with probability $\frac{r 2}{u}$. Hence, each relay node independently sends data with probability $\mu_{r}=\frac{r 2}{n m-2}$.

However, receiving and sending data is mutually exclusive events in cache queue of each relay node. The queue of the relay node can be seen as a continuous time queue with input rate $\lambda_{r}$ and service rate $\mu_{r}$, where $\frac{\lambda_{r}}{\mu_{r}}=\rho$. This holds for each relay node, the expected number of data is

$$
\bar{L}_{r}=\frac{\rho}{1-\rho} .
$$

Finally, when social parameter $\delta>3 / 2$, using Little's Theorem, we can get the average waiting time for S-D pairs-the network delay $D(n)$ is

$$
\begin{aligned}
D(n) & =\frac{\overline{L_{s}}+(n m-2) \overline{L_{r}}}{\lambda_{i}} \\
& =\frac{n m-1-\lambda_{i}}{\mu-\lambda_{i}} .
\end{aligned}
$$

For social parameter $\delta>3 / 2$, the network delay $D(n)$ is $\Theta(n m)$.

For social parameter $\delta \leq 3 / 2$, data is forward via a multi-hop path. Three kinds of transmissions are allowed: transmissions between source node and relay node, transmissions between relay node and relay node, transmissions between relay node and destination node. As illustrated in Figure 4, the process can be modeled as a open queuing network just as $\delta>3 / 2$.

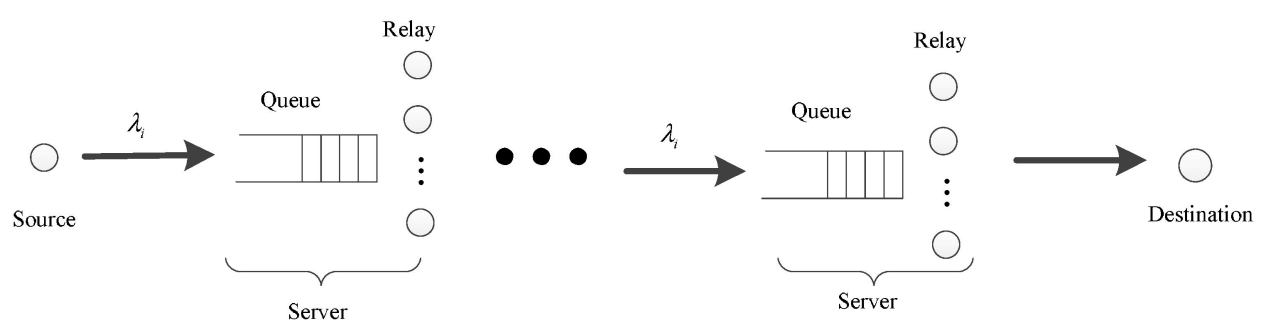

Figure 4. Queue in network for $\delta \leq 3 / 2$.

The super-cell where the source node is located is defined as the source super-cell; the super-cell where the relay node is located is defined as the relay super-cell; and the destination super-cell where the destination node is located is defined as the destination super-cell.

Using the same analytical method, we can get a same result in source super-cell. The source node have an expected number of data given by $\bar{L}_{s}=\frac{\rho\left(1-\lambda_{i}\right)}{1-\rho}$, where $\rho=\frac{\lambda_{i}}{\mu}$.

In relay super-cell and the destination super-cell, both the input and the output are Bernoulli stream of rate $\lambda_{i}$ because of a reversible queue. Data from this output process is transmitted to relay node. Because the total number of relay nodes in each super-cell is $n m-1$, data delivered to a relay node with probability $\frac{r 1}{u}$ equally.

Hence, each relay node independently receives data with probability $\lambda_{r}=\frac{\lambda_{i} r 1}{\mu(n m-1)}$. Because the total number of relay nodes in each super-cell is $n m-1$, the relay node is scheduled for data transmission to the destination node with probability $\frac{r 2}{u}$. Hence, each relay node independently sends data with probability $\mu_{r}=\frac{r 2}{n m-1}$.

Similarly, receiving and sending data is mutually exclusive events in cache queue of each relay node. which can be seen as a continuous time queue with input rate $\lambda_{r}$ and service rate $\mu_{r}$. 
This holds for all the relay super-cell, the expected number of data of the relay node is $\bar{L}_{r}=\frac{\rho}{1-\rho}$, where $\rho=\frac{\lambda_{r}}{\mu_{r}}$. Data is delivered along $\mathbb{S D}$ lines super-cells. To reach the destination node the average number of super-cells passing through by each source node is $\frac{E\{\mathcal{L}(S, D)\}}{\sqrt{m}}$.

Finally, summing the delay in all super-cell, we can get the total network delay

$$
\begin{aligned}
D(n) & =\frac{\sum \bar{L}}{\lambda_{i}} \\
& =\frac{\overline{L_{s}}+\sum_{i=1}^{h} \overline{L_{r}}}{\lambda_{i}} \\
& =\frac{1-\lambda_{i}}{\mu-\lambda_{i}}+\frac{E\{\mathcal{L}(S, D)\}}{\sqrt{m}}(n m-1) \frac{\bar{L}_{r}}{\lambda_{i}} .
\end{aligned}
$$

Substituting $E\{\mathcal{L}(S, D)\}$, we get the network delay in Theorem 3 .

Theorem 3. Based on the multi-hop relay and slots allocation scheme, the network delay $D(n)$ in large social-aware mobile ad hoc wireless networks can be expressed as

$$
D(n)= \begin{cases}\Theta(n \sqrt{m}) & 0 \leq \delta<1 \\ \Theta\left(\frac{n}{\log n} \sqrt{m}\right) & \delta=1 \\ \Theta\left(n^{2-\delta} \sqrt{m}\right) & 1<\delta<3 / 2 \\ \Theta\left(n^{1 / 2} \log n \sqrt{m}\right) & \delta=3 / 2 \\ \Theta(n m) & \delta>3 / 2 .\end{cases}
$$

\section{Discussion and Conclusion}

As illustrated in Theorems 1-3, both network capacity and network delay are affected by the social characteristics and node mobility. In this section we discuss and analyze the results.

Upper bound throughput of each node and achieved throughput of each node are listed in Table 2. From this table, we can see that the achieved throughput of each node differs from the upper bound throughput of each node only by a factor. Such discrepancies are consistent with the fact.

Table 2. Throughput capacity.

\begin{tabular}{cccccc}
\hline$\delta$ & $\delta<\mathbf{1}$ & $\delta=\mathbf{1}$ & $\mathbf{1}<\delta<3 / 2$ & $\delta=3 / 2$ & $\delta>3 / 2$ \\
\hline $\mathcal{T}$ & $O\left(\frac{\sqrt{m}}{\sqrt{\log n}}\right)$ & $O(\sqrt{\log n} \sqrt{m})$ & $O\left(\frac{n^{1-\delta} \sqrt{m}}{\sqrt{\log n}}\right)$ & $O\left(\frac{\sqrt{n} \sqrt{m}}{\log ^{3 / 2} n}\right)$ & $O\left(\frac{\sqrt{n} \sqrt{m}}{\log n}\right)$ \\
$\mu$ & $\Omega\left(\frac{1}{n \sqrt{m}}\right)$ & $\Omega\left(\frac{\log n}{n \sqrt{m}}\right)$ & $\Omega\left(\frac{1}{n^{2-\delta} \sqrt{m}}\right)$ & $\Omega\left(\frac{\sqrt{n} \sqrt{m}}{\log n}\right)$ & $\Omega\left(\frac{1}{\sqrt{n} \sqrt{m}}\right)$ \\
\hline
\end{tabular}

A common phenomenon can be seen from Table 2. Both the upper bound throughput of each node and the achieved throughput of each node increase with social characteristics $\delta$. Specifically, the value of achieved per-node throughput is between $\frac{1}{n \sqrt{m}}$ and $\frac{1}{\sqrt{n} \sqrt{m}}$. Minimum value of achieved per-node throughput is $\frac{1}{n \sqrt{m}}$, when $0 \leq \delta<1$. And the maximum value of achieved per-node throughput is $\frac{1}{\sqrt{n} \sqrt{m}}$ when $\delta>3 / 2$. The upper bound of per-node throughput shows the same change tendency.

From formula (8), we can seen that the greater value of social characteristics $\delta$, the shorter distance between S-D pair. With the increase of $\delta$, source node tends to select a closer destination node. This leads to traffic aggregation in large social-aware mobile ad hoc wireless networks. The parameter of social model depicts the degree of traffic aggregation. Traffic aggregation brings greater network capacity. 
When the parameter of node mobility $m$ is equal to $b(n)$, make the upper bound throughput of each node minimum. The the upper bound throughput of each node is $\frac{1}{\sqrt{n}}, \frac{\log n}{\sqrt{n}}, \frac{1}{n^{\delta-1 / 2}}, \frac{1}{\log n}$ and $\frac{1}{\sqrt{\log n}}$ respectively, when $\delta<1, \delta=1,1<\delta<3 / 2, \delta=3 / 2$ and $\delta>3 / 2$. The parameters of node mobility $m$ is equal to $\mathcal{O}$, make the upper bound throughput of each node maximum. In order to obtain higher throughput capacity, we need to increase $m$, which means a larger motion range.

Figure 5 exhibits that different scaling behaviors of network delay result from different values of social model parameter $\delta$. One can observe that for $\delta>3 / 2$ the best network delay can be achieved $\Theta(n m)$. Since $m \in(b(n), 1)$, it indicates that the network delay ranges from $\Theta(\sqrt{n})$ to $\Theta(n)$. As the parameter of social model becomes smaller, the network delay increases.

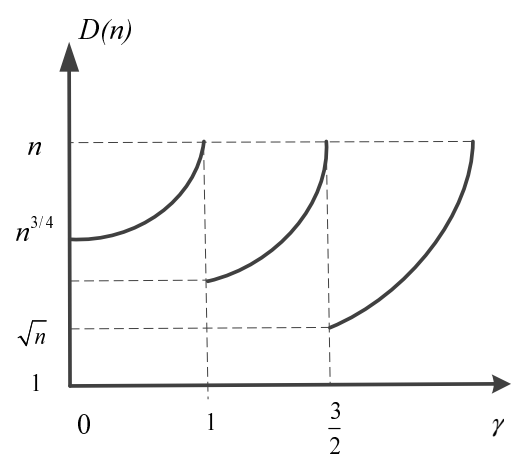

Figure 5. The network delay $D(n)$.

The relationship between network delay and throughput capacity is mutual contradiction. Bigger throughput capacity requires as many nodes as possible to send or receive data at any time, which makes the waiting queue increase. On the other hand, reducing the network delay requires waiting queue as short as possible, which makes throughput capacity smaller.

The throughput of each node is $\Theta\left(\frac{1}{\sqrt{n \log n}}\right)$ in static ad hoc wireless networks, meanwhile the network delay is negligible [5,6]; the throughput of each node is $\Theta(1)$ in mobile ad hoc wireless networks, with network delay $\Theta(n)$ [7]. Compared with existing works, we clearly show the gain on capacity, as well as on delay in large social-aware mobile ad hoc wireless networks. We propose a multi-hop scheduling and slots allocation scheme, and we try to improve the tradeoff between throughput capacity and network delay, as shown in Figure 6.

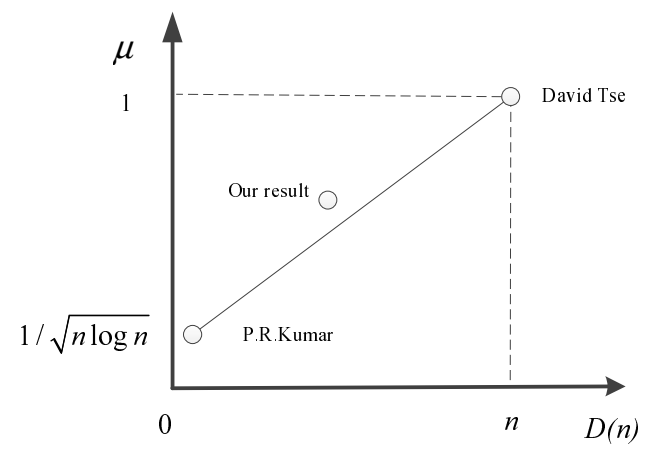

Figure 6. Throughput capacity and network delay tradeoff.

Our results provide a new insight on the performances of wireless ad hoc networks. It should be emphasized that our results are obtained under some idealistic assumptions. We will further extend our work to more realistic scenarios.

Author Contributions: Conceptualization, Y.Z., Y.S. and S.C.; Methodology, Y.Z.; Writing—original draft, Y.Z.; Writing-review and editing, Y.S. and S.C.; Project administration, Y.S.; Funding acquisition, Y.S. and S.C. All authors have read and agree to the published version of the manuscript. 
Funding: This research received no external funding.

Conflicts of Interest: The authors declare no conflict of interest.

\section{References}

1. Ghosh, A.; Maeder, A.; Baker, M.; Chandramouli, D. 5G Evolution: A View on 5G Cellular Technology Beyond 3GPP Release 15. IEEE Access 2019, 7, 127639-127651. [CrossRef]

2. Li, T.; Chen, Z.N. Shared-Surface Dual-Band Antenna for 5G Applications. IEEE Trans. Antennas Propag. 2019, 68, 1128-1133. [CrossRef]

3. Azees, M. Reply to Comments on dual Authentication and Key Management Techniques for Secure Data Transmission in Vehicular Ad Hoc Networks. IEEE Trans. Intell. Transp. Syst. 2019, 20, 3595-3595. [CrossRef]

4. Luo, Y.; Dang, J.; Song, Z.; Wang, B. Compressed sensing algorithm for neighbour discovery in mobile ad hoc networks. IET Commun. 2019, 13, 1781-1786. [CrossRef]

5. Gupta, P.; Kumar, P.R. The capacity of wireless networks. IEEE Trans. Inf. Theory 2000, 46, 388-404. [CrossRef]

6. Freris, N.M.; Kowshik, H.; Kumar, P.R. Fundamentals of Large Sensor Networks: Connectivity, Capacity, Clocks, and Computation. Proc. IEEE 2010, 98, 1828-1846. [CrossRef]

7. Grossglauser, M.; Tse, D. Mobility increases the capacity of ad-hoc wireless networks. In Proceedings of the IEEE Twentieth Annual Joint Conference of the IEEE Computer and Communications Societies INFOCOM 2001, Anchorage, AK, USA, 22-26 April 2001; Volume 3, pp. 1360-1369. [CrossRef]

8. Franceschetti, M.; Dousse, O.; Tse, D.N.C.; Thiran, P. Closing the Gap in the Capacity of Wireless Networks Via Percolation Theory. IEEE Trans. Inf. Theory 2007, 53, 1009-1018. [CrossRef]

9. Peraki, C.; Servetto, S.D. On the Maximum Stable Throughput Problem in Random Networks with Directional Antennas. In Proceedings of the 4th ACM International Symposium on Mobile Ad Hoc Networking \& Computing, MobiHoc '03, Annapolis, MD, USA, 1-3 June 2003; ACM: New York, NY, USA, 2003; pp. 76-87. [CrossRef]

10. Huang, W.; Wang, X. Throughput and delay scaling of general cognitive networks. In Proceedings of the 2011 Proceedings IEEE INFOCOM, Shanghai, China, 10-15 April 2011; pp. 2210-2218. [CrossRef]

11. Jiang, C.; Shi, Y.; Hou, Y.T.; Kompella, S. On the Asymptotic Capacity of Multi-Hop MIMO Ad Hoc Networks. IEEE Trans. Wirel. Commun. 2011, 10, 1032-1037. [CrossRef]

12. Li, X.Y. Multicast Capacity of Wireless Ad Hoc Networks. IEEE/ACM Trans. Netw. 2009, 17, 950-961. [CrossRef]

13. Li, X.Y.; Liu, Y.; Li, S.; Tang, S. Multicast Capacity of Wireless Ad Hoc Networks Under Gaussian Channel Model. IEEE/ACM Trans. Netw. 2010, 18, 1145-1157. [CrossRef]

14. Lin, X.; Sharma, G.; Mazumdar, R.R.; Shroff, N.B. Degenerate delay-capacity tradeoffs in ad-hoc networks with Brownian mobility. IEEE Trans. Inf. Theory 2006, 52, 2777-2784. [CrossRef]

15. Ciullo, D.; Martina, V.; Garetto, M.; Leonardi, E. Impact of correlated mobility on delay-throughput performance in mobile ad hoc networks. IEEE/ACM Trans. Netw. (TON) 2011, 19, 1745-1758. [CrossRef]

16. Lee, K.; Kim, Y.; Chong, S.; Rhee, I.; Yi, Y.; Shroff, N.B. On the Critical Delays of Mobile Networks Under Levy Walks and Flights. IEEE/ACM Trans. Netw. 2013, 21, 1621-1635. [CrossRef]

17. Liu, J.; Kato, N.; Ma, J.; Sakano, T. Throughput and Delay Tradeoffs for Mobile Ad Hoc Networks With Reference Point Group Mobility. IEEE Trans. Wirel. Commun. 2015, 14, 1266-1279. [CrossRef]

18. Luo, Z.; Cui, Y.; Wang, X.; Luo, H. On the Throughput and Delay in Ad Hoc Networks With Human Mobility. IEEE Trans. Commun. 2015, 63, 2273-2287. [CrossRef]

19. Neely, M.J.; Modiano, E. Capacity and delay tradeoffs for ad hoc mobile networks. IEEE Trans. Inf. Theory 2005, 51, 1917-1937. [CrossRef]

20. Zemlianov, A.; de Veciana, G. Capacity of ad hoc wireless networks with infrastructure support. IEEE J. Sel. Areas Commun. 2005, 23, 657-667. [CrossRef]

21. Zhang, G.; Xu, Y.; Wang, X.; Tian, X.; Liu, J.; Gan, X.; Yu, H.; Qian, L. Multicast Capacity for VANETs with Directional Antenna and Delay Constraint. IEEE J. Sel. Areas Commun. 2012, 30, 818-833. [CrossRef]

22. Mori, H. Fault Tolerance of Small World Network Architecture. In Proceedings of the 2018 Sixth International Symposium on Computing and Networking Workshops, Takayama, Japan, 27-30 November 2018; pp. 344-349. [CrossRef] 
23. Luo, D.; Qiu, T.; Deonauth, N.; Zhao, A. A small world model for improving robustness of heterogeneous networks. In Proceedings of the 2015 IEEE Global Conference on Signal and Information Processing (GlobalSIP), Orlando, FL, USA, 14-16 December 2015; pp. 849-852. [CrossRef]

24. Hou, R.; Cheng, Y.; Li, J.; Sheng, M.; Lui, K. Capacity of Hybrid Wireless Networks With Long-Range Social Contacts Behavior. IEEE/ACM Trans. Netw. 2017, 25, 834-848. [CrossRef]

25. Wei, Z.; Wu, H.; Yuan, X.; Huang, S.; Feng, Z. Achievable Capacity Scaling Laws of Three-Dimensional Wireless Social Networks. IEEE Trans. Veh. Technol. 2018, 67, 2671-2685. [CrossRef]

26. Qin, Y.; Jia, R.; Zhang, J.; Wu, W.; Wang, X. Impact of Social Relation and Group Size in Multicast Ad Hoc Networks. IEEE/ACM Trans. Netw. 2016, 24, 1989-2004. [CrossRef]

(C) 2020 by the authors. Licensee MDPI, Basel, Switzerland. This article is an open access article distributed under the terms and conditions of the Creative Commons Attribution (CC BY) license (http:/ / creativecommons.org/licenses/by/4.0/). 